\title{
Translating artificial intelligence into clinical practice
}

\author{
Duoru Lin, Haotian Lin \\ State Key Laboratory of Ophthalmology, Zhongshan Ophthalmic Center, Sun Yat-sen University, Guangzhou, China \\ Correspondence to: Professor Haotian Lin, MD, PhD. Xian Lie South Road \#54, Guangzhou, China. Email: gddlht@aliyun.com.
}

Submitted Sep 15, 2019. Accepted for publication Nov 19, 2019.

doi: $10.21037 /$ atm.2019.11.110

View this article at: http://dx.doi.org/10.21037/atm.2019.11.110

With increasing reports of breakthroughs of artificial intelligence (AI) in medicine in recent years, the U.S. Food and Drug Administration (FDA) permitted marketing of the first medical AI-based device to screen and monitor diabetic retinopathy in April 2018 (1), indicating an expected near boom of clinical use of AI in health care. However, the appropriate application pattern still requires further exploration regarding how to apply AI in real-world clinical practice to translate this exciting technological advance into meaningful clinical impacts.

For this breakthrough technology, it is necessary to rationally recognize the advantages and insufficiencies of present medical AI. Because the reported outperformance of AI mainly came from laboratories with high-quality and well-structured data (2), the initial real-world application of $\mathrm{AI}$ is recommended for solving well-defined problems and must be performed under strict quality control for safety. Furthermore, the high efficiency and stability of computer-driven AI are especially suitable for large-scale screenings for diseases with characteristic morphological changes, with a relatively low risk of medical accidents and a substantial value of easing the contradiction between the overwhelming need for healthcare and the limited medical resources in China. To maximize the effects of $\mathrm{AI}$ in health care under safety protocols, a practical application pattern to alleviate the medical supply and need contradiction (AMSNC) is proposed. In the AMSNC pattern, a network connecting thousands of mobile terminals and dozens of primary hospitals to one tertiary hospital forms a basic AI alliance. The primary hospitals are equipped with AI techniques and are responsible for the primary diagnosis of common diseases and large-scale disease screening from mobile terminals or disease screening sites; while the tertiary hospital is set as a referral and quality control center.
Cataracts are the leading cause of visual impairment worldwide (3) and show characteristic morphological changes (lens opacity) and a relatively low risk of medical accidents, which is well suitable for the exploration of translating AI technique into real-world clinical practice. An ophthalmic management AI platform connected to 65 community hospitals has been established at the Zhongshan ophthalmic center (ZOC) (4), a representative ophthalmic tertiary hospital in China, aiming at improving the eye care capability of primary hospitals and alleviating the workload of doctors in tertiary hospitals. This highquality medical resource also extends to families for home monitoring, in that residents can obtain authoritative medical opinions at home by uploading their ocular photos to the cloud platform using smartphones. Patients with an uncertain diagnosis or those requiring further treatments will be referred to the specifically established ophthalmic AI clinic in the ZOC (the first ophthalmic AI clinic in China) for final diagnosis and further treatment. Up till now, nearly 40,000 ocular images were received from the mobile terminals and community hospitals, and more than 1,000 referable cataractous patients were identified (5).

Although the exploration of the most appropriate application pattern of AI in ophthalmic health care has been taken in China, a set of optimal mechanisms for $\mathrm{AI}$ in clinical use, including the ethical and legal frameworks for data sharing, clinical responsibility, and multi-department collaboration, still require further improvement for a wider range of applications.

\section{Acknowledgments}

Funding: This study was funded by the National Key R\&D Program of China (2018YFC0116500); the Key Research Plan for the National Natural Science Foundation of 
China in Cultivation Project (91846109); the Science and Technology Planning Projects of Guangdong Province (2018B010109008); China Postdoctoral Science Foundation (2018M640860, 2019T120773), Fundamental Research Funds for the Central Universities (18ykpy33), and Fundamental Research Funds of the State Key Laboratory of Ophthalmology (2018-2019).

\section{Footnote}

Provenance and Peer Review: This article was commissioned by the Guest Editors (Haotian Lin and Limin Yu) for the series "Medical Artificial Intelligent Research" published in Annals of Translational Medicine. The article was sent for external peer review organized by the Guest Editors and the editorial office.

Conflicts of Interest: Both authors have completed the ICMJE uniform disclosure form (available at http://dx.doi. org/10.21037/atm.2019.11.110). The series "Medical Artificial Intelligent Research" was commissioned by the editorial office without any funding or sponsorship. HL served as the unpaid Guest Editor of the series. DL has no other conflicts of interest to declare.

Ethical Statement: The authors are accountable for all aspects of the work in ensuring that questions related to the accuracy or integrity of any part of the work are

Cite this article as: Lin D, Lin H. Translating artificial intelligence into clinical practice. Ann Transl Med 2020;8(11):715. doi: 10.21037/atm.2019.11.110 appropriately investigated and resolved.

Open Access Statement: This is an Open Access article distributed in accordance with the Creative Commons Attribution-NonCommercial-NoDerivs 4.0 International License (CC BY-NC-ND 4.0), which permits the noncommercial replication and distribution of the article with the strict proviso that no changes or edits are made and the original work is properly cited (including links to both the formal publication through the relevant DOI and the license). See: https://creativecommons.org/licenses/by-nc-nd/4.0/.

\section{References}

1. FDA permits marketing of artificial intelligence-based device to detect certain diabetes-related eye problems. Cited 2018 Jul 16. Available online: https://www.fda. gov/NewsEvents/Newsroom/PressAnnouncements/ ucm604357.htm

2. The Lancet. Artificial intelligence in health care: within touching distance. Lancet 2018;390:2739.

3. Epidemiology of cataract. Lancet 1982;1:1392-3.

4. Zheng BX. Welcome doc. AI to primary hospitals. Guangzhou Daily. Cited 2018 Jul 17. Available online: http://gzdaily.dayoo.com/pc/html/2018-06/22/ content_2_2.htm?v=C8

5. Wu X, Huang Y, Liu Z, et al. Universal artificial intelligence platform for collaborative management of cataracts. Br J Ophthalmol 2019;103:1553-60. 